\title{
Hypoechoic nodule structure increases non-diagnostic rate of thyroid fine needle aspiration biopsy
}

\author{
(1)Zeynep Çetin \\ Amasya University, Faculty of Medicine, Department of Internal Medicine, Division of Endocrinology and Metabolism, Amasya, Turkey
}

Cite this article as: Çetin Z. Hypoechoic nodule structure increases non-diagnostic rate of thyroid fine needle aspiration biopsy. J Health Sci Med 2021; 4(6): 886-891.

\begin{abstract}
Aim: Thyroid fine needle aspiration biopsy (FNAB) performed with ultrasonography is an effective method in diagnosing thyroid cancer. Nevertheless, some of them have non-diagnostic results. The aim is to investigate the factors that affect nondiagnostic result.

Material and Method: FNABs of 361 nodules of 361 patients were analyzed retrospectively. The patients were divided into two groups as fine needle aspiration biopsy result with and without non-diagnostic. The groups were compared according to demographic, clinical and sonographic data.

Results: Non-diagnostic rate was $14.4 \%$ in all biopsies. There was no difference in terms of age, gender, previous thyroid surgery history, smoking history, aspirin use, Hashimoto's thyroiditis, toxic nodule. Sonographic findings of the thyroid glands and nodules were similar. Only the echogenicity of the nodule was found to affect the non-diagnostic result ( $\mathrm{p}=0.015)$. In the post hoc analysis, the difference was found to be caused by hypoechoic nodules (32.7\% versus 15.5$)$. It was observed that the significant relationship found in univariate logistic regression analysis ( $p=0.009$, OR: 3.227, CI: 1.334-7.803) continued in multivariate analysis ( $\mathrm{p}=0.024$, OR: 3.175, CI: 1.163-8.668).
\end{abstract}

Conclusion: Only hypoechoic echogenicity increases the risk of non-diagnostic rate. Other factors do not increase the nondiagnostic rate.

Keywords: Thyroid fine needle aspiration biopsy, ultrasonography, non diagnostic, hypoechogenicity

\section{INTRODUCTION}

The frequency of thyroid cancer continues to increase with each passing year. With the proliferation of ultrasonography (USG), nodular thyroid disease has been detected more frequently. The most appropriate method for making the necessary surgical decision and avoiding unnecessary surgery and related complications is fine needle aspiration biopsy (FNAB) $(1,2)$. FNAB is an easy, inexpensive and effective diagnostic method. It is more effective when performed with USG. It has a 95\% negative, $97-99 \%$ positive predictive value. FNAB indication is based on the sonographic features of the thyroid nodule. FNAB should be performed in the case of hypoechogenicity, irregular edge structure, microcalcification, ratio of anterior-posterior diameter to transverse diameter $>1$, and central vascularity (3).

FNAB assessment is done according to the Bethesda system and the results are given in 6 categories; benign, non-diagnostic (ND), indeterminate atypia (AUS)/ indeterminate follicular lesion (AUFL), suspicious for follicular neoplasia or follicular neoplasia (FN), suspicious cytology for malignancy and malignancy (4). In the best series, the ND rate is $2-16 \%(5,6)$. Fifty five- $74 \%$ of biopsies are reported as benign, $2-5 \%$ as malignant, $2-18 \%$ as AUS/AUFL, $2-25 \%$ as FN and $1-6 \%$ as suspicious for malignancy (6).

In order to FNAB to be sufficient, there must be at least 6 groups of follicular cells containing at least 10 wellpreserved epithelial cells in one area. It is reported as ND, which does not meet these criteria. It is recommended to repeat FNAB, and if ND comes again, surgery or close follow-up is recommended. While the estimated malignancy risk of the nodule with a biopsy ND is $1-4 \%$, the actual risk of malignancy of this nodule is 9-32\% (5). A nodule whose biopsy is 2 times ND has a $25 \%$ risk of being malignant (7). Therefore, surgical and medical follow-up decision should be made carefully in nodules whose biopsy is ND. 
Studies investigating the factors affecting FNAB adequacy can be found in the literature. Different causes such as advanced patient age, nodule heterogeneity (8), aspirin (9), radioactive iodine therapy (10) were found to be related to ND. In this study, it was aimed to investigate the factors affecting ND biopsy results in a large sample group.

\section{MATERIAL AND METHOD}

\section{Ethical Approval}

The study was carried out with the permission of Amasya University Non-interventional Clinical Researchs Ethics Committee (Date: 02.05.2019, Decision No: 05-26). All procedures were in accordance with the ethical standards of the institutional research committee and with the 1964 Helsinki declaration and its later amendments or comparable ethical standards. The subjects have given their written informed consent. This study does not contain animals.

\section{Patient Selection}

Between January 1, 2018 and January 1, 2019, 361 patients who underwent FNAB were included. These biopsies were performed by an endocrinologist experienced in terms of USG and FNAB. One FNAB result of each patient was included in the study. Consent was obtained from the participants before and after the FNAB. Demographic, laboratory, ultrasound and FNAB data of the participants were obtained from the hospital information processing system. The age, gender, smoking status, aspirin usage, previous thyroid surgery was recorded. According to thyroid autoantibodies and USG, which patients had Hashimoto's thyroiditis (HT) was detected. Thyroid scanningwasperformed bytheendocrinologistwith thyroid ultrasonography device (Aloka, Mollsfeld, Meerbusch, Germany). Thyroid gland and nodule volumes were calculated according to the formula of anterior-posterior diameter $\mathrm{x}$ transverse diameter $\mathrm{x}$ longitudinal diameter $\mathrm{x}$ 0.52 (11). Whether the nodule is thyrotoxic in hyperthyroid patients was recorded based on thyroid scintigraphy. Biopsy Indications and Results

FNAB was performed according to the size $(>1 \mathrm{~cm})$, content (solid), echogenicity (hypoechoic), edge structure (irregular), vascularity (central), calcification (microcalcification), anterior-posterior diameter/ transverse diameter ratio $(>1)$ of the thyroid nodules (12). Local anesthesia was not applied before biopsy. FNAB was made by aspiration method by 22 -gauge needle. During aspiration, the USG probe was turned from the transverse position to the longitudinal position, the needle was inserted into the nodule at a right angle to the neck, the needle was moved 5-6 times to the anteriorposterior and lateral sides. The material was spread to the slides by the endocrinologist at the bedside. Slides were delivered to the pathology department after being dried in the air. FNAB results were categorized by experienced cytopathologists according to the Bethesda system (4).

\section{Statistical Analysis}

Statistical analyzes were made in IBM SPSS for Windows Version 22.0. Numerical variables were summarized with mean \pm standard deviation. Categorical variables were indicated by number and percentage. The chisquare test or Fisher's exact test was used to determine whether there was any difference between the groups in terms of categorical variables. The Kolmogorov Smirnov test, standard deviation/mean ratio, skewness/kurtosis measures, histogram and detrended normal graphics were used to determine whether the numeric variables showed normal distribution, and the homogeneity of the variance was examined by the Levene test. Differences between two independent groups in terms of numerical variables; In the case of parametric test assumptions, t-test was used for independent groups. In the absence of parametric test assumptions, Mann Whitney U test was used. Post hoc analysis was used to compare more than 2 groups. Univariate and multivariate logistic regression analyses were applied to determinate independent predictors affecting ND result. The significance level was taken as $\mathrm{p}<0.05$.

\section{RESULTS}

The results of the FNABs are as shown in Table 1. ND rate was $14.4 \%$ among all biopsies. The most reported category was benign, with a rate of $67.6 \%$ (Table 1 ).

All participants were divided into two groups according to whether the FNAB result was ND or not. Data on age, advanced age ( $>65)$, gender, previous history of thyroid surgery, smoking status, use of aspirin, presence of HT are shown in Table 2. No significant difference was found between the two groups (Table 2).

Thyroid USG features of all participants are given in Table 3. There was a significant difference in nodule echogenicity between the groups $(\mathrm{p}=0.015)$. Post hoc analysis was performed to determine which subtype caused the difference. The difference was caused by hypoechoic subgroup by applying Bonferoni correction and adjusted residual (Table 3 ).

\begin{tabular}{|lc|}
\hline Table 1. FNAB results & \\
\hline Total, n & 361 \\
Non-diagnostic, n (\%) & $52(14.4)$ \\
Benign, n (\%) & $244(67.6)$ \\
AUS/AUFL ${ }^{\text {a }}$ n (\%) & $50(13.9)$ \\
Follicular neoplasia, n (\%) & $4(1.1)$ \\
Malignant, n (\%) & $1(0.2)$ \\
Suspicious for malignancy, n (\%) & $10(2.8)$ \\
\hline a: AUS/AUFL: unspecified atypia/indeterminate follicular lesion & \\
\hline
\end{tabular}




\begin{tabular}{|c|c|c|c|c|}
\hline Parameters & Total $(n=361)$ & Non-ND ${ }^{a}$ group $(n=309)$ & ND $^{\text {a }}$ group $(n=52)$ & $\mathbf{p}$ \\
\hline Age, mean \pm std dev & $53.03 \pm 11.78$ & $52.66 \pm 11.96$ & $54.79 \pm 10.35$ & 0.227 \\
\hline Age $>65$, n (\%) & $58(16)$ & $49(15.9)$ & $9(17.3)$ & 0.792 \\
\hline Gender, n (\%) & & & & 0.417 \\
\hline Male & $75(20.8)$ & $62(20.1)$ & $17(25)$ & \\
\hline Female & $286(79.2)$ & 247 (79.9) & $39(75)$ & \\
\hline Previous thyroid surgery, $\mathrm{n}(\%)$ & $25(6.9)$ & $22(7.1)$ & $3(12)$ & 0.784 \\
\hline Smoking, n (\%) & $60(16.6)$ & $48(15.5)$ & $12(23.1)$ & 0.176 \\
\hline Aspirin, n (\%) & $42(11.6)$ & $37(12)$ & $5(9.6)$ & 0.624 \\
\hline $\mathrm{HT}^{\mathrm{b}}, \mathrm{n}(\%)$ & $92(25.5)$ & $83(26.8)$ & $9(17.3)$ & 0.184 \\
\hline $\mathrm{TSH}$, mean \pm std dev & $1.84 \pm 7.94$ & $1.89 \pm 8.56$ & $1.59 \pm 1.71$ & 0.896 \\
\hline
\end{tabular}

\begin{tabular}{|c|c|c|c|c|}
\hline Parameter & Total $(n=361)$ & Non-NDa group $(n=309)$ & $N^{a}$ group $(n=52)$ & $\mathbf{P}$ \\
\hline Right thyroid volume, $\mathrm{mL}$ & $20.403 \pm 15.016$ & $20.429 \pm 15.272$ & $20.250 \pm 13.525$ & 0.697 \\
\hline Left thyroid volume, $\mathrm{mL}$ & $18.580 \pm 14.020$ & $18.381 \pm 14.290$ & $19.760 \pm 12.354$ & 0.202 \\
\hline Istmus, mm & $5.71 \pm 3.99$ & $5.64 \pm 4$ & $6.14 \pm 3.95$ & 0.348 \\
\hline Parenchym & & & & 0.891 \\
\hline Homogeneous & $21(5.8)$ & $17(5.5)$ & $4(7.7)$ & \\
\hline Mild heter ${ }^{b}$ & $181(50.1)$ & $157(50.8)$ & $24(46.2)$ & \\
\hline Moderate heter ${ }^{b}$ & $113(31.3)$ & $96(31.1)$ & $17(32.7)$ & \\
\hline Advanced heter ${ }^{b}$ & $46(12.7)$ & $39(12.6)$ & $7(13.5)$ & \\
\hline Nodule noc, n (\%) & & & & 0.950 \\
\hline Solitary & $89(28.1)$ & $76(24.6)$ & $13(25)$ & \\
\hline MNGd & $272(71.9)$ & $233(75.4)$ & $39(75)$ & \\
\hline Nodule size, n (\%) & & & & 0.332 \\
\hline$<10 \mathrm{~mm}$ & $15(4.1)$ & $11(3.6)$ & $4(7.7)$ & \\
\hline $10.01-39.99 \mathrm{~mm}$ & $312(86.4)$ & $270(87.4)$ & $42(80.8)$ & \\
\hline$>40 \mathrm{~mm}$ & $37(9.5)$ & $28(9)$ & $6(11.5)$ & \\
\hline A-P diame/T diamf & & & & 0.142 \\
\hline$<1$ & 345 (95.6) & $293(94.9)$ & $52(100)$ & \\
\hline$>1$ & $16(4.4)$ & $16(5.1)$ & $0(0)$ & \\
\hline Content,n (\%) & & & & 0.113 \\
\hline Solid & $158(43.7)$ & $130(42.1)$ & $28(53.8)$ & \\
\hline Mixed & $203(56.3)$ & $179(57.9)$ & $24(46.2)$ & \\
\hline Echogenity,n(\%) & & & & 0.015 \\
\hline Isoechoic & $91(25.2)$ & $82(26.5)$ & $9(17.3)$ & \\
\hline Hypoechoic & $65(18)$ & $48(15.5)$ & $17(32.7)$ & \\
\hline Hyperechoic & $13(3.6)$ & $10(3.2)$ & $3(5.8)$ & \\
\hline Spongiform & $192(53.2)$ & $169(54.8)$ & $23(53.2)$ & \\
\hline Edge, n (\%) & & & & 0.393 \\
\hline Smooth & $270(74.8)$ & $230(74.4)$ & $40(76.9)$ & \\
\hline Irregular & $61(16.9)$ & $53(17.2)$ & $8(15.4)$ & \\
\hline Undefined & $30(8.3)$ & $26(8.4)$ & $4(7.7)$ & \\
\hline Halo, n (\%) & & & & 0.284 \\
\hline Absent & $325(90)$ & 275 (89) & $50(96.2)$ & \\
\hline Present & $36(10)$ & $34(11)$ & $2(3.8)$ & \\
\hline Calcification,n (\%) & & & & 0.885 \\
\hline Absent & 303 (83.9) & $261(84.5)$ & $42(80.8)$ & \\
\hline Microcalcification & $10(2.7)$ & $9(2.9)$ & $1(1.9)$ & \\
\hline Macrocalcification & $28(7.7)$ & $22(7.1)$ & $6(11.5)$ & \\
\hline Micro+macrocalc & $7(1.9)$ & $6(1.9)$ & $1(1.9)$ & \\
\hline Eggshell calc. & $13(3.8)$ & $11(3.6)$ & $2(3.8)$ & \\
\hline Vascularity, n (\%) & & & & 0.365 \\
\hline Absent & $224(62)$ & $194(62.8)$ & $30(57.7)$ & \\
\hline Peripheral & $108(29.9)$ & $89(28.8)$ & $19(36.5)$ & \\
\hline Central & $3(0.8)$ & $2(0.6)$ & $1(1.9)$ & \\
\hline Periph+central & $26(7.3)$ & $24(7.8)$ & $2(3.8)$ & \\
\hline Toxic nodule, $\mathrm{n}(\%)$ & $50(13.9)$ & $41(13.3)$ & $9(17.3)$ & 0.435 \\
\hline
\end{tabular}


Table 4. Univariate and multivariate regression analysis

\begin{tabular}{|c|c|c|c|c|c|c|}
\hline \multirow[t]{2}{*}{ Parameter } & \multicolumn{3}{|c|}{ Univariate } & \multicolumn{3}{|c|}{ Multivariate } \\
\hline & $\mathbf{P}$ & OR $^{\mathbf{a}}$ & $95 \% \mathrm{CI}$ & $\mathbf{P}$ & OR & $95 \% \mathrm{CI}^{\mathrm{b}}$ \\
\hline Nodule echogenicity & $0.009^{*}$ & 3.227 & $1.334-7.803$ & $0.024^{*}$ & 3.175 & $1.163-8.668$ \\
\hline Nodule size (>1-3.99) & $0.037^{\star}$ & 0.303 & $0.098-0.930$ & 0.077 & 0.311 & $0.085-1.137$ \\
\hline
\end{tabular}

In univariate logistic regression analysis, nodule echogenicity and size were found to have a significant effect on the ND result ( $p=0.009$ and $p=0.037$ ). In the multivariate analysis, while the effect of echogenicity on FNAB remained ( $\mathrm{p}=0.024$, OR: 3.175 , CI: $1.163-8.668$ ), the effect of the nodule size was not detected ( $\mathrm{p}=0.077$, OR: 0.311, CI: 0.085-1.137) (Table 4).

\section{DISCUSSION}

In this study, the fine needle aspiration biopsy results performed by the endocrinologist in 1 year were investigated and the factors affecting the ND result were investigated. According to the results of the analysis, it was found that only hypoechogenity was increasing the likelihood of ND results.

In the study, $14.4 \%$ of total biopsies were determined as ND. This rate is similar to the literature since the probability of obtaining ND results is $2-16 \%(5,6)$. Results in other categories (except for those malignant and suspected of malignancy) are similar to the literature (6). The experience of the endocrinologist performing the biopsies provided the ND rate to be similar to the literature, and the experience is an important factor affecting the success of the biopsy (13).

Patients were divided into two groups as those with and without ND FNABs, and they were compared according to age, gender, previous history of thyroid surgery, smoking, presence of HT, thyroid USG findings.

Inci and his colleagues (8) retrospectively examined the results of FNABs performed by a radiologist (not endocrinologist) of 270 individuals in 2 years, ND rate was $31.9 \%$ and they found that advanced patient age is a factor that increases the risk of ND. Based on this study, we compared the groups in terms of age. The average age of the first study was 46 and 53 in our study. In the first study, the number of geriatric patients was not specified. In our case, there are roughly $16 \%$ geriatric patients. Contrary to the first study, we did not find a significant difference between the groups in terms of both the average age and the distribution of geriatric population, and we can say that age is not a risk factor for ND result. ND rate of the first study was markedly high, which may have led to such a conclusion about age.

The gender distribution was also similar in terms of the two groups. As in many studies, although the number of women who underwent FNAB in our center was significantly higher than men, the effect of gender on ND outcome was not detected. For example, in the study of Ozel et al. (14), gender distribution in ND and non-ND groups is similar.

Postoperative recurrent nodular thyroid disease is seen in $2-42 \%$ in different series in patients without total thyroidectomy (15). Onal et al. (16) found that, subtotal thyroid surgery increases the risk of ND outcome by causing fibrosis and scar formation. We also considered our patients' history of thyroid surgery. The patients who had previous thyroid surgery were $6.9 \%$ and consisted of recurrent MNG cases with FNAB indication in the residual thyroid gland. There was no significant difference in the distribution of these patients into groups $(7.1 \%$ versus $12 \%, p=0.784)$. The conclusion drawn from this is that, unlike the previous study, having a previous thyroid surgery does not increase the risk of ND. However, since the number of patients with operated recurrent nodular goiter in our study was lower compared to other studies (123 patients versus 25 in total), more participatory studies are needed to support this result.

Since smoking exposure has been shown to worsen Graves' ophthalmopathy, studies have been conducted to demonstrate the relationship between smoking and thyroid. Although these studies have shown that smoking increases the risk of nodule formation and thyroid cancer $(17,18)$, there are also studies showing that it is ineffective $(19,20)$. In the literature, as we have determined, there are no studies investigating the effect of cigarette exposure on ND biopsy results. For this reason, we also considered the smoking history of the patients. When the distribution of the patients who ex-smokers and still smokers was compared between the two groups, no significant relationship was found $(\mathrm{p}=0.176$, table 2). However, we did not elaborate on cigarette exposure (duration, amount, passive smoking, etc.). Therefore, more detailed studies to support this finding will help enlighten the issue.

$\mathrm{HT}$ is the most common inflammatory thyroid disease. The main diagnosis is made histopathologically, but in daily practice, HT is diagnosed according to thyroid autoantibodies and USG findings (18). According to the studies, the success of FNAB decreases in the presence of $\mathrm{HT}$ and it increases the diagnosis ND and AUS/AUFL 
$(21,22)$. In our study, while HT was detected in $25.5 \%$ of all patients, we could say that HT did not increase the risk of ND since there was no significant difference between the groups in terms of HT.

Toxic nodules previously thought to be protective for thyroid cancer, more FNABs are being performed today and it has been found to carry a risk of malignancy as much as non-toxic nodular goiter (23). Baser et al. (24) examined FNAB results of toxic and non-toxic nodules and showed that they had similar ND ratios. In our study, toxic nodules with FNAB were also included because they had sonographically risky features. The distribution of toxic nodules was similar between the groups, and it was determined that the toxic nodule had no effect on the ND result.

Studies investigating the efficacy/adequacy of thyroid FNAB were mostly based on sonographic findings. In our study, we compared both the general thyroid USG findings of the patients and the features of the nodules undergoing FNAB. There was no difference between the two groups in terms of thyroid gland size, parenchymal structure and number of nodules. The nodule size, the ratio of anteriorposterior diameter to transverse diameter, nodule content, echogenicity, margin arrangement, vascularity and calcification content were compared and only the nodule echogenicity was effective on the ND result. This difference was also shown by post hoc analysis that it was caused by hypoechoic echogenicity (table 3 ). In both univariate and multivariate logistic regression analyzes, hypoechogenity has been shown to affect the ND biopsy result. So, it was shown in our study that only hypoechoic echogenicity contributed to the ND result. Inci et al. (8) stated that heterogeneous echogenicity leads to more ND results, which may result from the hemorrhagic and necrotic content of the nodule. Ozel et al. (14) concluded that needle size is effective in ND result, and sonographic features of nodule have no effect. Moon and colleagues (25) found hypoechogenity as an independent factor for ND, as in our study. There are many other studies showing that more ND results are obtained in hypoechoic nodules $(26,27)$. The possible reason is that due to the hypoechoic nodules being more fibrotic, sufficient material cannot be taken with fine needle aspiration (26). While cystic content increased the risk of ND in some studies $(13,28)$, it was shown that it did not pose any risk in some (27). In our study, cystic content did not increase the risk of ND. No relation was found between size, vascularity, calcification (especially macrocalcification) and ND, as in many other studies $(8,13,27)$.

Consequently, according to our study, the only factor that adversely affects the FNAB adequacy is the nodule hypoechogenity. Other demographic, clinical and sonographic features do not affect biopsy adequacy.
Our study provided homogeneity, covering a shorter period of time (1 year), by the same expert (1 endocrinologist), using the same USG, the same needle (22 gauge) and the same technique (aspiration). Although it covers 1 year, the number of patients (361) undergoing FNAB is quite high. We believe that this is a detailed study since it includes both demographic, clinical and USG findings.

Our study had limitations, too. We included only 1 biopsy result of 1 nodule of each patient, we did not include the results of re-biopsy. For this reason, we were unable to answer the question of whether the rate of ND will change with re-biopsy. The sample size of the study is also one of the factors limiting the study (361 biopsies performed in 1 year by 1 endocrinologist were examined.). Considering how common thyroid nodules are, analyzes with larger numbers of subjects are needed.

In some studies, the thickness of the needle used for FNAB was also investigated. The general recommendation is to make FNAB with a fine needle.12,28 We performed all biopsies with a 22 - gauge needle and we did not compare different needles, so we could not comment on this.

\section{CONCLUSION}

Sonographic imaging and fine needle aspiration biopsy have an important role in the evaluation of thyroid nodules. The most important factor affecting biopsy adequacy is the echogenicity of the nodule. Performing the biopsy with USG, reduces non-diagnostic results.

\section{ETHICAL DECLARATIONS}

Ethics Committee Approval: The study was carried out with the permission of Amasya University Noninterventional Clinical Researchs Ethics Committee (Date: 02.05.2019, Decision No: 05-26).

Informed Consent: All patients signed the free and informed consent form.

Referee Evaluation Process: Externally peer-reviewed.

Conflict of Interest Statement: The author has no conflicts of interest to declare.

Financial Disclosure: The author declared that this study has received no financial support.

Author Contributions: The author daclared (design, execution, and analysis) that all stages of the work were done by the author herself.

\section{REFERENCES}

1. Le AR, Thompson G, Hoyt BJ. Thyroid Fine-needle aspiration biopsy: an evaluation of its utility in a community setting. J Otolaryngol Head Neck Surg 2015; 44: 12-6. 
2. Huang LY, Lee YL, Chou P, Chiu WY, Chu D. Thyroid fine-needle aspiration biopsy and thyroid cancer diagnosis: a nationwide population-based study. PLoS ONE 2015; 10: e0127354.

3. Olson MT, Zelger MA. Thyroid fine-needle aspiration and cytological diagnosis. In: Jameson JL, De Groot LJ, editors. Endocrinology adult and pediatric. 7th edition Philadelphia: Elsevier Saunders 2016: 1417-22.

4. Ali SZ, Cibas ES. The Bethesda system for reporting thyroid cytopathology. definitions, criteria and explanatory notes. New York-USA: Springer, 2010.

5. Bongiovanni M, Spitale A, Faquin WC, Mazzucchelli L, Baloch ZW. The Bethesda system for reporting thyroid cytopathology: a meta-analysis. Acta Cytol 2012; 56: 333-9.

6. Luu MH, Fischer AH, Pisharodi L, Owens CL. Improved preoperative definitive diagnosis of papillary thyroid carcinoma in FNAs prepared with both ThinPrep and conventional smears compared with FNAs prepared with ThinPrep alone. Cancer Cytopathol 2011; 119: 68-73.

7. Moon HJ, Kwak JY, Choi YS, Kim EK. How to manage thyroid nodules with two consecutive nondiagnostic results on ultrasonography-guided fine-needle aspiration. World J Surg 2012: 586-92.

8. Inci MF, Ozkan F, Yuksel M, Salk I, Sahin M. The effects of sonographic and demographic features and needle size on obtaining adequate cytological material in sonographyguided fine-needle aspiration biopsy of thyroid nodules. Endocrine 2013 43: 424-9.

9. Khan TS, Sharma E, Singh B, et al. Aspirin Increases the Risk of Nondiagnostic Yield of Fine-Needle Aspiration and Biopsy of Thyroid Nodules. Eur Thyroid J 2018; 7: 129-32.

10.Ziemiańska K, Kopczyński J, Kowalska A. Repeated nondiagnostic result of thyroid fine-needle aspiration biopsy. Contemp Oncol (Pozn) 2016; 20: 491-5.

11. Shabana W, Peeters E, Verbeek P, Osteaux MM. Reducing intraobserver variation in thyroid volume calculation using a new formula and technique. Eur J Ultrasound 2003; 16: 207-10.

12. Haugen BR, Alexander EK, Bible KC, et al. 2015 American Thyroid Association Management Guidelines for Adult Patients with Thyroid Nodules and Differentiated Thyroid Cancer. THYROID 2016; 26: 1-133

13.Penín M, Martín MA, Millán BS, Garcia J. Learning curve of thyroid fine-needle aspiration biopsy. Endocrinol Diabetes Nutr 2017; 64: 539-43.

14. Ozel D, Ozel BD, Ozkan F. Potential causes for obtaining non-diagnostic results from fine needle aspiration biopsy of thyroid nodules. Radiol Med 2016; 121: 510-4.

15. Gutaj, NS, Ziółkowska P, Sowiński J. Recurrent goiter: risk factors, patient quality of life, and efficacy of radioiodine therapy. Pol Arch Intern Med 2019; 129: 22-27.

16. Onal ED, Saglam F, Sacikara M, Ersoy R, Guler G, Cakır B. The diagnostic accuracy of thyroid nodule fine-needle aspiration cytology following thyroid surgery: a case-control study. Endocr Pathol 2014; 25: 297-301.

17. Cho YA, Kim J. Thyroid cancer risk and smoking status: a metaanalysis. Cancer Causes Control 2014; 25: 1187-95.

18. Kim SJ, Kim MJ, Yoon SG, et al. Impact of smoking on thyroid gland: dose-related effect of urinary cotinine levels on thyroid function and thyroid autoimmunity. Sci Rep 2019; 9: 1-6.

19. Karatoprak C, Kartal I, Kayatas K. Does smoking affect thyroid gland enlargement and nodule formation in iodine-sufficient regions? Ann Endocrinol (Paris) 2012; 73: 542-5.

20. Wu G, Zou D, Cai H, Liu Y. Ultrasonography in the diagnosis of Hashimoto's thyroiditis. Front Biosci (Landmark Ed) 2016; 21 1006-12.
21.Meng C, Hinkle, LE, Wang W, Su D, Li X. Hashimoto's thyroiditis elicits decreased diagnostic efficacy of thyroid nodule ultrasoundguided fine needle aspiration. Int J Clin Exp Pathol 2019; 12: 3474-82.

22. Gao L, Ma B, Zhou L. The impact of presence of Hashimoto's thyroiditis on diagnostic accuracy of ultrasound-guided fineneedle aspiration biopsy in subcentimeter thyroid nodules: a retrospective study from FUSCC. Cancer Med 2017; 6: 1014-22.

23. Smitt JJ, Chen X, Schneider DF. Toxic nodular goiter and cancer: a compelling case for thyroidectomy. Ann Surg Oncol 2016; 20: 1336-40.

24. Baser H, Topaloglu O, Bilginer MC. Are cytologic and histopathologic features of hot thyroid nodules different from cold thyroid nodules? Diagn Cytopathol 2019; 47: 898-903.

25. Moon HJ, Kwak JY, Kim EK, Kim MJ. Ultrasonographic characteristics predictive of nondiagnostic results for fine-needle aspiration biopsies of thyroid nodules. Ultrasound Med Biol 2011; 37: 549-55

26. Chen SJ, Yu SN, Tzeng JE, et al. Characterization of the major histopathological components of thyroid nodules using sonographic textural features for clinical diagnosis and management. Ultrasound Med Biol 2009; 35: 201- 8 .

27.Degirmenci B, Haktanir A, Albayrak R, et al. Sonographically guided fine-needle biopsy of thyroid nodules: the effects of nodule characteristics, sampling technique, and needle size on the adequacy of cytological material. Clin Radiol 2007; 62: 798-803.

28. Choi YS, Hong SW, Kwak JY, Moon HJ, Kim EK. Clinical and ultrasonographic findings affecting nondiagnostic results upon the second fine needle aspiration for thyroid nodules. Ann Surg Oncol 2012; 19: 2304-09. 